\title{
Toolbox for Interval Type-2 Fuzzy Logic Systems
}

\author{
Mohsen Zamani ${ }^{1}$ Hossein Nejati $^{2}$ Amin T. Jahromi $^{3}$ \\ Ali Reza Partovi ${ }^{4}$ Sadegh H. Nobari $^{2}$ Ghasem N. Shirazi $^{1}$ \\ ${ }^{1}$ Electrical and Computer Engineering Dept, National University \\ of Singapore, Singapore \\ zamani@nus.edu.sg; naddafzadeh@nus.edu.sg \\ ${ }^{2}$ School of Computing, National University of Singapore, Singapore \\ nejati@nus.edu.sg; snobari@comp.nus.edu.sg \\ ${ }^{3}$ Electrical and Electronic Engineering School, Nanyang \\ Technological University, Singapore \\ amin0005@ntu.edu.sg \\ ${ }^{4}$ Electrical Engineering Dept, Alame Mohaddeseh Noori \\ Technical University, Noor, Iran \\ ali.partovi@gmail.com
}

\begin{abstract}
Type-2 systems has been becoming the focus of research in the field of fuzzy logic in recent years. Comparing with type-1 systems, type-2 fuzzy systems are more complex and relatively more difficult to understand and implement. We developed an interactive graphical user interface (GUI) based toolbox, MFLS tool, for interval type-2 fuzzy logic system. This paper presents MFLS toolbox. Moreover, the versatility of the software is demonstrated via an prediction problem.
\end{abstract}

Keywords: Interval Type-2 Fuzzy logic System; Toolbox

\section{Introduction}

Fuzzy logic has obtained attention of researchers for last couple of decades. It has opened new horizons both in the academia and the industry site. Fuzzy logic proposed by Zadeh(1965) has found his way during ages in many different applications. Although, conventional fuzzy systems (FSs) or so called type-1 FSs is capable of handling input uncertainties, it is not adequate to handle all types of uncertainties associated with knowledge-based systems. Thus, [20] introduced type-2 FSs as an extension to his first theory for type1. On the other hand, type-2 theory is capable of handling 4 major type of uncertainties involved with type-1 FS [15] .

Type-1 membership function are exact defined, whereas type- 2 membership function has one more degree of freedom which suites them for uncertain environments but higher degree of freedom brings computation complexity to type-2 fuzzy logic systems(FLSs). Type-2 is much computationally intensive comparing to its type-1 counterpart. Despite their computation burden, type- 2 has been applied to various type of application such as, signal processing [13, 12], pattern recognition $[9,8]$, time-series forecasting $[10,16]$, decision making [17], finance[4], wire- 
less communication [14], noise cancellation [5], system identification [11], neural network [18], power engineering [1], control [7, 6]. Although, type-2 has been emerging in different branch of science, its popularity is still not comparable to its conventional counterpart. Type- 2 has not extended its domain due to following factors:

1) Type-2 fuzzy set are computationally more complex that their conventional counter part.

2) There is still a lack of useful toolboxes to facilitate the use of type- 2 .

This paper presents development and design of a GUI and a command line programming toolbox for construction, edition and observation of interval type-2 Fuzzy inference systems. The MFLS tool, is an environment for implementing and designing of a interval type- 2 FLS (IT2FLS). MFLS covers all phases of the IT2FLS from first phase till the last. The MFLS best qualities such as flexibility which enables the user to add new file, and user friendly which makes it suitable for versatile range of user from beginner to advance. Moreover, it contains some function which implement the inference engine as black box.

In Section 2 some topics on IT2FLS is demonstrated, in Section 3 developed toolbox is presented; in Section MFLS is exploited for general approximation problem. Finally, conclusion are stated in Section 5 .

\section{Interval Type-2 Set and Systems}

Interval type-2 fuzzy set is a special case of general type- 2 set in which there is no fuzziness in its third dimension and secondary grade is just equal to identity. (1), (2) present type-2 fuzzy set for continuous and discrete domain, respectively.

$$
\begin{gathered}
\grave{A}=\int_{x \in X}\left[\int_{v \in J_{x}} 1 / v / x\right] \quad J_{x} \subseteq[0,1] \\
\grave{A}=\sum_{i=1}^{N}\left[\sum_{v \in J_{x}}[1 / v]\right] / x_{i}
\end{gathered}
$$

It is well known [15] that an interval set $\mu_{\grave{A}}(x)$ can be fully expressed based on upper $\bar{\mu}_{\grave{A}}(x)$ and lower membership function $\underline{\mu}_{\hat{A}}(x)$. Some of the most fundamental type-2 membership function are listed below:

1. Gaussian with uncertain mean

$$
\begin{aligned}
& \bar{f}_{\grave{A}}(x)=\left\{\begin{array}{cc}
N(m 1, \sigma ; x) & x<m 1 \\
1 & m 1 \leq x \leq m 2 \\
N(m 2, \sigma ; x) & x>m 2
\end{array}\right. \\
& \underline{f}_{\grave{A}}(x)=\left\{\begin{array}{cc}
N(m 2, \sigma ; x) & x \leq \frac{m 1+m 2}{2} \\
N(m 1, \sigma ; x) & x>\frac{m 1+m 2}{2}
\end{array}\right.
\end{aligned}
$$

2. Gaussian with uncertain deviation

$$
\begin{aligned}
& \bar{f}_{\grave{A}}(x)=N\left(m, \sigma_{2} ; x\right) \\
& \underline{f}_{\grave{A}}(x)=N\left(m, \sigma_{1} ; x\right)
\end{aligned}
$$

3. Triangular membership function with uncertain mean

$$
\begin{gathered}
\bar{f}_{\grave{A}}(x)=\left\{\begin{array}{cc}
0 & x<l_{1} \\
\frac{x-l_{1}}{p_{1-l}} & l_{1} \leq x<p_{1} \\
1 & p_{1} \leq x \leq p_{2} \\
\frac{r_{2}-x}{r_{2}-p_{2}} & p_{2}<x \leq r_{2} \\
0 & x>r_{2}
\end{array}\right. \\
\underline{f}_{\grave{A}}(x)=\left\{\begin{array}{cc}
0 & x<l_{2} \\
\frac{x-l_{2}}{p_{2}-l_{2}} & x \leq \frac{r_{1}\left(p_{2}-l_{2}\right)+l_{2}\left(r_{1}-p_{1}\right)}{\left(p_{2}-l_{2}\right)+\left(r_{1}-p_{1}\right)} \\
\frac{r_{2}-x}{r_{2}-p_{2}} & x>\frac{r_{1}\left(p_{2}-l_{2}\right)+l_{2}\left(r_{1}-p_{1}\right)}{\left(p_{2}-l_{2}\right)+\left(r_{1}-p_{1}\right)} \\
0 & x>r_{2}
\end{array}\right.
\end{gathered}
$$

In fuzzy reasoning the consequence set can be concluded even if the antecedent set does not completely satisfy so, fuzzy reasoning is not as precise as conventional logic. The IT2FLS is depicted in Figure 1. 


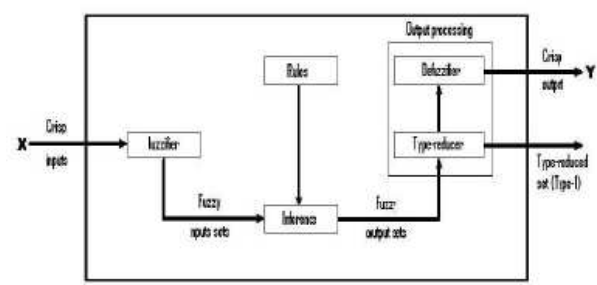

Fig. 1: IT2FLS inference structure

\section{MFLS Toolbox}

Mamdani IT2FLS is designed and implemented in MFLS toolbox. Intersection operators such as product and minimum and union operators like maximum or summation. Moreover, MFLS allows the user to either choose center of sum, center of set or centroid as desired type reduction procedure. The package exploits KarnikMendel algorithm to obtain the type reduced set. This package offers the user flexibility for handling computation time by adjusting quantization step. MFLS has two main parts a GUI based part and a MATLAB part. Visual C\# based GUI helps the user to set all the parameter and then exports set parameters in to MATLAB environment as a structure variable. For instance, Figure 2 depicted the front panel of MFLS, and Figures 3, 4, 5 depict MFLS windows for some of well known type-2 membership function.

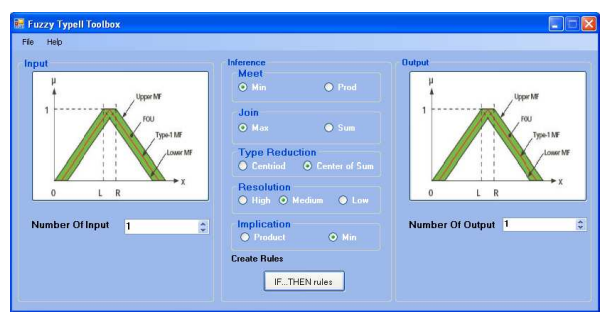

Fig. 2: MFLS main panel

In this part we demonstrate a required procedure for implementing FS

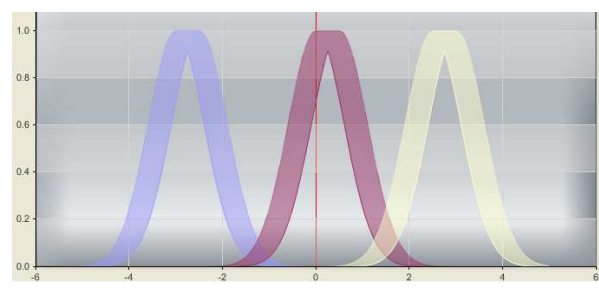

Fig. 3: Gaussian membership function with uncertain mean

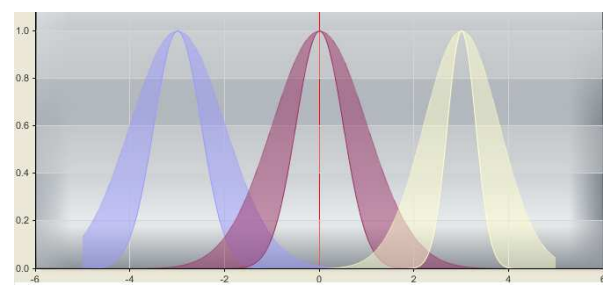

Fig. 4: Gaussian membership function with uncertain deviation

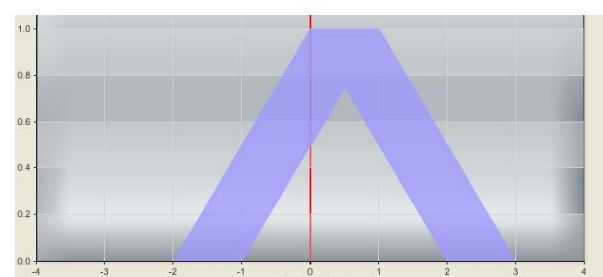

Fig. 5: Triangular membership function with uncertain mean

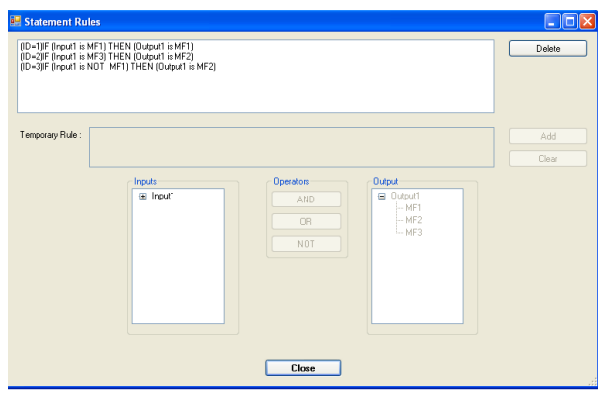

Fig. 6: Rule Base in MFLS

with MFLS. Firstly, the cardinality of antecedent and consequent must be set. Secondly, fuzzy rules must be created, a 
set of fuzzy rules are shown in Figure 6. Thirdly, for exploiting the designed IT2FLS in MATLAB, user needs to export the IT2FLS in to MATLAB mfile using file \export system menu. The result of this part is structure variable which reflects the designed system. The block diagram in Figure 7 demonstrates the linking variable between GUI and MATLAB. The user can modify the structure for his own personal purpose.

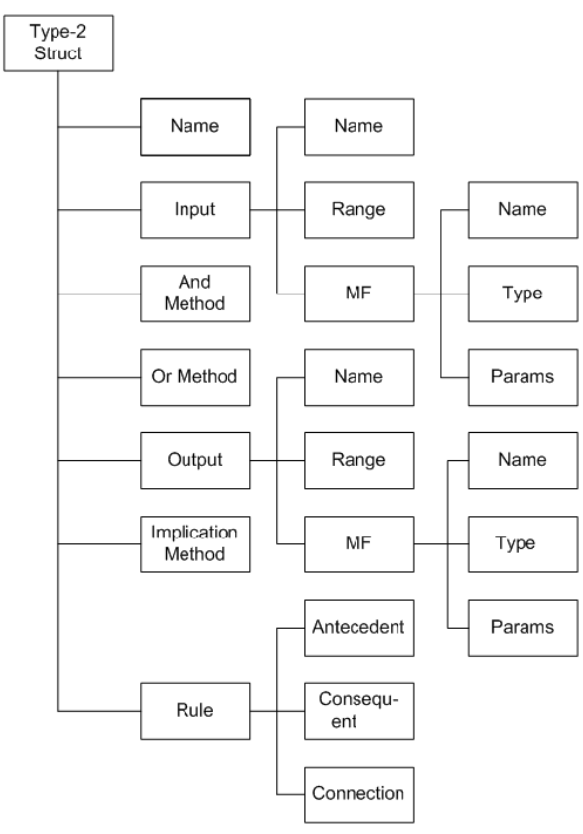

Fig. 7: Structure variable which links the GUI and MATLAB

On the other hand, some mfiles are responsible for linkage between GUI and MATLAB software. This makes the problem easy for user. Some of the function are as follows plottype $2 . \mathrm{m}$, kmim.m, inference.m and etc. also, for sake of simplicity all the required functions are integrated in to one function called type 2 fls.m which helps user to deal with IT2FLS as black box. This way user does not need to worry about detail material and user just need to concern about top level issues. The process of design based on MFLS is depicted in Figure 8.

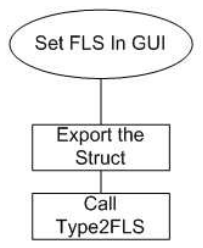

Fig. 8: FS design sequence with MFLS

\section{Application}

In this section a general approximation problem is introduced and the MFLS toolbox is exploited for solving this problem. There is approximation based on noisy data [15]. The objective is to approximate $y=100-x^{2}$ where $x \in[-10,10]$. The only access is just available to noisy measurement. For any test point $x_{i}, y_{i}$ bounds to $\left[y_{l i}, y_{u i}\right]$ so, it means that FS relies on non-crisp data value. The Gaussian membership function with uncertain mean is desired for the outputs. There is no uncertainty involved with inputs. Thus, input membership function is considered to be type-1. The input and output ranges are $x \in[-10,10], y \in[-190,190]$, respectively. The singleton fuzzification and minimum, product and center of sum are assigned for intersection operator, product operator and type reduction method, respectively.

All antecedent sets assumed to be Gaussian function and their parameters are listed in Table 1 . On the other hand, the consequent sets are Gaussian membership function with uncertain mean. The consequent parameters are reported in Table 2. The FS use the simple rule base as following:

$$
\text { IF } x \text { is } A_{i} \text { THEN } y \text { is } C_{i} \text {. }
$$




\begin{tabular}{|l|c|c|}
\hline & mean & deviation \\
\hline$A_{1}$ & -10 & 1.25 \\
$A_{2}$ & -7.5 & 1.25 \\
$A_{3}$ & -5 & 1.25 \\
$A_{4}$ & -2.5 & 1.25 \\
$A_{5}$ & 0 & 1.25 \\
$A_{6}$ & 2.5 & 1.25 \\
$A_{7}$ & 5 & 1.25 \\
$A_{8}$ & 7.5 & 1.25 \\
$A_{9}$ & 10 & 1.25 \\
\hline
\end{tabular}

Table 1: Antecedent set parameters

\begin{tabular}{|l|c|c|c|}
\hline & mean1 & mean2 & deviation \\
\hline$C_{1}$ & -7.79 & 6.49 & 40 \\
$C_{2}$ & 34.72 & 52.93 & 40 \\
$C_{3}$ & 66.12 & 84.1 & 40 \\
$C_{4}$ & 84.93 & 101.75 & 40 \\
$C_{5}$ & 93.09 & 109.95 & 40 \\
$C_{6}$ & 88.02 & 103.53 & 40 \\
$C_{7}$ & 65.37 & 84.32 & 40 \\
$C_{8}$ & 34.14 & 50.85 & 40 \\
$C_{9}$ & -9.62 & 9.62 & 40 \\
\hline
\end{tabular}

Table 2: Consequent set parameters

The design steps are mentioned in Figure 8 and resultant output is depicted in Figure 9. Although,in this example there were not precise access to value of $y$ and it varies in large scale, the type-2 FS approximates the function with close approximation.

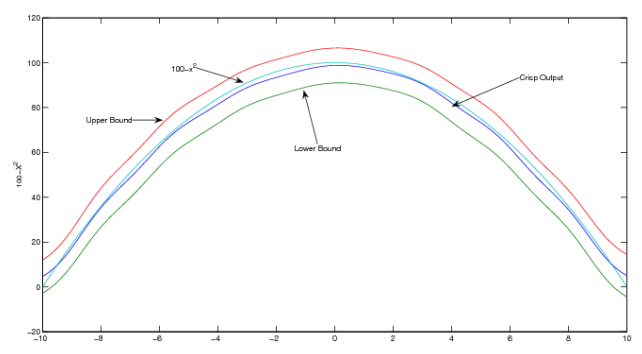

Fig. 9: Output response

\section{Conclusion}

In this paper MFLS toolbox, a toolbox for type-2 FS was presented and versatility of toolbox is demonstrated via an general approximation example. Our future work is to improve MFLS by adding new functions, developing more advanced GUI and integrating intelligent learning technique such as genetic algorithm and neural network.

\section{References}

[1] J. R. Agero and A. Vargas, Using type-2 fuzzy logic systems to infer the operative configuration of distribution networks, in Proceedings IEEE Power Engineering Society General Meeting, pages 2379-2386, June 2005.

[2] S. Auephanwiriyakul, A. Adrian, and J.M. Keller, Type 2 fuzzy set analysis in management surveys. in Procce IEEE International Conference on Fuzzy Systems, vol. 2, pages 13211325, 2002.

[3] S. Auephanwiriyakul, A. Adrian, and J.M. Keller, Type 2 fuzzy set analysis in management surveys. in Procce IEEE International Conference on Fuzzy Systems, vol. 2, pages 1321$1325,2002$.

[4] P. Baguley, T. Page, V. Koliza, and P. Maropoulos, Time to market prediction using type-2 fuzzy sets. Journal of Manufacturing Technology Msanagement, 17(4):513-520, 2006.

[5] O. Castillo and P. Melin, Adaptive noise cancellation using type-2 fuzzy logic and neural networks. in IEEE Conference on Cybernetics and Intelligent Systems, vol. 2, pages 13511355, 2004.

[6] J. Figuero, J. Posada, M. Melgarejo, and S. Rojas, A type-2 fuzzy controller for tracking mobile objects in the context of robotic soccer games.in 
Proceedings 2005 IEEE International Conference on Fuzzy Systems, pages 359-364, May 2005.

[7] H. Hagras. A hierarchical type-2 fuzzy logic control architecture for autonomous mobile robots. IEEE Transactions on Fuzzy Systems, 12(4):524539, August 2004.

[8] P. Herman, G. Prasad, and T. M. McGinnity, Investigation of the type-2 fuzzy logic approach to classification in an EEG-based brain-computer interface. In Proceedings of the Annual International Conference-IEEE Engineering in Medicine and Biology Society, pages 5354-5357, 2005.

[9] Jia Zeng and Zhi-Qiang Liu. Type-2 fuzzy sets for handling uncertainty in pattern recognition, in Procceding of FUZZ-IEEE, pages 6597-6602, Vancouver, Canada, July 2006.

[10] N. N. Karnik and J. M. Mendel, Applications of type-2 fuzzy logic systems to forecasting of timeseries, Information Sciences, vol. 120, pages 89-111, 1999.

[11] C. H. Lee, Y.C. Lin, and W. Y. Lai, Systems identification using type2 fuzzy neural network (type-2 FNN) systems. in Proceedings of IEEE International Symposium on Computational Intelligence in Robotics and Automation. Computational Intelligence in Robotics and Automation for the New Millennium, vol. 3, pages 1264-1269, 2003.

[12] Q. Liang and J. M. Mendel, Equalization of nonlinear time-varying channels using type-2 fuzzy adaptive filters, IEEE Transactions on Fuzzy Systems, 2000.

[13] Q. Liang and J.M. Mendel, Modeling MPEG VBR video traffic using type2 fuzzy logic systems, In Granular Computing: An Emerging Paradigm, Springer-Verlag, 2000.

[14] Q. Liang and L. Wang, Sensed signal strength forecasting for wireless sensors using interval type-2 fuzzy logic system. in Proceedings 2005 IEEE International Conference on Fuzzy Systems, pages 25-30, Reno, NV, May 2005.

[15] J. M. Mendel, Uncertain RuleBased Fuzzy Logic Systems Introduction and New Directions, Englewood Cliffs, NJ: Prentice-Hall, 2001.

[16] J. M. Mendel, Uncertainty, fuzzy logic, and signal processing, Signal Proccessing, vol. 80, pages 913-933, 2000.

[17] T. Ozen and J.M. Garibaldi. Effect of type-2 fuzzy membership function shape on modelling variation in human decision making. In Proceedings 2004 IEEE International Conference on Fuzzy Systems, vol. 2, pages 971976, 2004.

[18] F.C.H. Rhee and C. Hwang, An interval type-2 fuzzy perceptron. in IEEE International Conference on Fuzzy Systems, FUZZ-IEEE vol. 2, pages 1331-1335, 2002.

[19] H. Shu and Q. Liang, Wireless sensor network lifetime analysis using interval type-2 fuzzy logic systems.in Proceedings 2005 IEEE International Conference on Fuzzy Systems, pages 19-24, May 2005.

[20] Zadeh, L.A,The concept of a linguistic variable and its application to approximate reasoning, Parts 1,2 , and 3 Information Sciences, 1975, 8:199249, 8:301-357, 9:43-80. 